\title{
Slowly Rotating Black Holes with Nonlinear Electrodynamics
}

\author{
S. H. Hendi ${ }^{1,2}$ and M. Allahverdizadeh ${ }^{3}$ \\ ${ }^{1}$ Physics Department and Biruni Observatory, College of Sciences, Shiraz University, Shiraz 71454, Iran \\ ${ }^{2}$ Center for Excellence in Astronomy \& Astrophysics of Iran (CEAAI-RIAAM), P.O. Box 55134-441, Maragha, Iran \\ ${ }^{3}$ Korea Institute of Science and Technology, Center for Functional Connectomics, Seoul 136-791, Republic of Korea \\ Correspondence should be addressed to S. H. Hendi; hendi@shirazu.ac.ir
}

Received 13 May 2014; Accepted 15 July 2014; Published 20 August 2014

Academic Editor: Rong-Gen Cai

Copyright (c) 2014 S. H. Hendi and M. Allahverdizadeh. This is an open access article distributed under the Creative Commons Attribution License, which permits unrestricted use, distribution, and reproduction in any medium, provided the original work is properly cited. The publication of this article was funded by SCOAP ${ }^{3}$.

\begin{abstract}
We study charged slowly rotating black hole with a nonlinear electrodynamics (NED) in the presence of cosmological constant. Starting from the static solutions of Einstein-NED gravity as seed solutions, we use the angular momentum as the perturbative parameter to obtain slowly rotating black holes. We perform the perturbations up to the linear order for black holes in 4 dimensions. These solutions are asymptotically AdS and their horizon has spherical topology. We calculate the physical properties of these black holes and study their dependence on the rotation parameter $a$ as well as the nonlinearity parameter $\beta$. In the limit $\beta \rightarrow \infty$, the solution describes slowly rotating AdS type black holes.
\end{abstract}

\section{Introduction}

The Reissner-Nordström (RN) solution is the static solution of the Einstein-Maxwell gravity. These solutions are asymptotically flat and their horizon has spherical topology. This black hole can be characterized by its mass and electric charge and the geometry of the RN black hole is singular at the origin of the radial coordinate. So, people were searching to construct a good regular alternative for it. For several decades a great deal of attention has been focused on some gravitating NED as regular generalizations of the RN solution of the Einstein-Maxwell gravity, in which the most popular example is the gravitating Born-Infeld (BI) theory [1-4]. On the other hand, theories such as the theory of black objects, with a logarithmic electrodynamic Lagrangian coupled to gravity, have been studied in an attempt to remove some of the singularities associated with a charged black hole [5]. The divergence of the energy-momentum tensor was successfully removed, although the spacetime still exhibited a curvature singularity, albeit of a weaker variety. Some other NED models supporting asymptotically Schwarzschildlike solutions have been also considered in the literature [5-7]. One can look for regular solutions with nonlinear electromagnetic fields of the Born-Infeld type [8-12].
On the other hand, exact solutions for charged static black holes with NED have been studied by many authors (see, e.g., $[5,11,13-18]$ ). This showed that the presence of the NED has important consequences for the black hole properties which motivate us to investigate charged black holes with NED, for instance, various limitations of the linear electrodynamics $[19,20]$, clarification of the selfinteraction of virtual electron-positron pairs [21-23], and description of radiation propagation inside specific materials [24-27]. Moreover, from astrophysical point of view, one finds the effects of NED become indeed quite important in superstrongly magnetized compact objects, such as pulsars, and particular neutron stars (some examples include the so-called magnetars and strange quark magnetars) [28-30]. Also, NED modifies in a fundamental basis the concept of gravitational redshift and its dependency of any background magnetic field as compared to the well-established method introduced by standard general relativity. In addition, it was recently shown that NED objects can remove both of the big bang and black hole singularities [31-36]. Furthermore, it was shown that the nonlinearity may change the geometric properties of the black hole horizon(s). For example, unlike charged black holes in Einstein-Maxwell gravity, there is a new situation of black hole horizon in the presence of nonlinear electrodynamics (see [11] for more details). Finally 
we should note that although NED theories have complicated calculation, there is no objection against these theories and they may lead to new consequences in the future, as Plebanski in 1968 said: [37]: "If in recent times the interest in NED cannot be said to be very popular, it is not due to the fact that one could rise some serious objections against this theory. It is simply rather difficult in its mathematical formulation, what causes that it is very unlikely to derive some concrete results in closed form."

However, generalizing these static black holes to the rotating solutions is not easy due to the complexity of the equations. This forces us to restrict ourselves to the limit of slow rotation. Slowly charged rotating black hole solutions in higher dimensions with a single rotation parameter have been investigated by many authors [38-46]. Using perturbation theory, they have introduced the rotation parameter as a perturbative parameter and solved the equations of motion up to the linear order of the angular momentum parameter. Here, we use the same approach. We started from the static charged black holes with NED in antide Sitter (AdS) spacetime [11] and then considered the effect of adding a small angular momentum to the solutions. We discarded any terms involving $a^{2}$ or higher powers in $a$, where $a$ is the rotation parameter. Finally we study the physical properties of these black holes. In particular, we have shown that the perturbative parameter $a$ and the nonlinearity parameter $\beta$ do not change the value of gyromagnetic ratio of these slowly rotating black holes.

The rest of the paper is organized as follows. In the next section, we give a brief review of the Einstein- $\Lambda$ field equations in the presence of NED. In Section 3, we present 4-dimensional slowly rotating charged black hole solutions in the presence of two new classes of NED. Then, we obtain mass, electric charge, temperature, entropy, angular momentum, and gyromagnetic ratio of the solutions. We finish our paper with some concluding remarks.

\section{Basic Field Equations}

The Lagrangian of Einstein gravity in the presence of negative cosmological constant, $\Lambda$, with a NED is

$$
\mathfrak{E}=R-2 \Lambda+\mathscr{L}_{\mathrm{NED}},
$$

where $R$ is the Ricci scalar and $\mathscr{L}_{\text {NLED }}$ can be selected as a one of two new classes of NED [10-12], namely, Exponential form of NED (ENED) and Logarithmic form of NED (LNED), whose Lagrangians are

$$
L(\mathscr{F})= \begin{cases}\beta^{2}\left(\exp \left(-\frac{\mathscr{F}}{\beta^{2}}\right)-1\right), & \text { ENED, } \\ -8 \beta^{2} \ln \left(1+\frac{\mathscr{F}}{8 \beta^{2}}\right), & \text { LNED, }\end{cases}
$$

where $\beta$ is the nonlinearity parameter and $\mathscr{F}=F_{\mu \nu} F^{\mu \nu}$ is Maxwell invariant. It is easy to show that in the limit $\beta \rightarrow \infty$ (weak field limit), the mentioned $L(\mathscr{F})$ 's reduce to the Lagrangian of the standard Maxwell field

$$
L(\mathscr{F}) \longrightarrow-\mathscr{F}+O\left(\mathscr{F}^{2}\right) .
$$

Using the Euler-Lagrange equation, we can obtain gravitational field equations as well as electromagnetic ones with the following explicit form:

$$
\begin{gathered}
G_{\mu \nu}+\Lambda g_{\mu \nu}=\frac{1}{2} g_{\mu \nu} L(\mathscr{F})-2 L_{\mathscr{F}} F_{\mu \lambda} F_{\nu}^{\lambda}, \\
\partial_{\mu}\left(\sqrt{-g} L_{\mathscr{F}} F^{\mu \nu}\right)=0,
\end{gathered}
$$

where $L_{\mathscr{F}}=d L(\mathscr{F}) / d \mathscr{F}$.

\section{4-Dimensional Slowly Rotating Charged Black Holes}

Here, we look for the slowly rotating nonlinear charged black hole solutions. Inspection of the 4-dimensional Kerr and Kerr-Newman spacetimes shows that the only term in the metric that changes to the first order of the angular momentum parameter $a$ is $g_{t \phi}$. In other words, using series expansion for slowly rotating Kerr-Newman spacetime, one obtains

$$
\begin{aligned}
d s^{2}= & -G(r) d t^{2}+\frac{d r^{2}}{G(r)} \\
& +2 a F(r) K(\theta) d t d \phi+r^{2}\left(d \theta^{2}+\sin ^{2} \theta d \phi^{2}\right),
\end{aligned}
$$

where the functions $G(r), F(r)$, and $K(\theta)$ are known functions in Einstein-Maxwell gravity and $a$ is a parameter associated with its angular momentum. In this paper, we take into account metric (6), to solve (4) and (5) to first order in the angular momentum parameter $a$. Using metric (6), one finds that the consistent one-form gauge potential is

$$
A=h(r)[d t+a K(\theta) d \phi] .
$$

After some algebraic calculations, we find that (5) leads to two independent differential equations. One of these equations is free of metric function with the following explicit form:

$$
e_{1}= \begin{cases}E^{\prime}(r)+\frac{2 E(r)}{r} \frac{\beta^{2}}{4 E^{2}(r)+\beta^{2}}=0, & \text { ENEF, } \\ E^{\prime}(r)-\frac{2 E(r)}{r} \frac{E^{2}(r)-4 \beta^{2}}{E^{2}(r)+4 \beta^{2}}=0, & \text { LNEF }\end{cases}
$$

and the following solution:

$$
E(r)= \begin{cases}\frac{q}{r^{2}} \exp \left[\frac{-L_{W}}{2}\right], & \text { ENEF, } \\ \frac{2 q}{r^{2}(\Gamma+1)}, & \text { LNEF, }\end{cases}
$$

where $E(r)=h^{\prime}(r)$, prime denotes the first derivative with respect to $r, \Gamma=\sqrt{1+\left(q^{2} /\left(\beta^{2} r^{4}\right)\right)}$, and $q$ is an integration constant which is the electric charge of the black holes. In addition, $L_{W}=\operatorname{Lambert} W\left(4 q^{2} /\left(\beta^{2} r^{4}\right)\right)$ which satisfies 
Lambert $W(x) \exp [\operatorname{Lambert} W(x)]=x$. (for more details, see $[47,48])$. Now, we expand the function $E(r)$ for large $r(r \gg 1)$ to investigate the asymptotic behavior of the electromagnetic field

$$
\left.E(r)\right|_{\text {Larger }}=\frac{q}{r^{2}}-\frac{\chi q^{3}}{4 \beta^{2} r^{6}}+O\left(\frac{q^{5}}{\beta^{4} r^{10}}\right),
$$

where $\chi=1$ and 8 for LNED and ENED, respectively. This equation shows that for large values of $r$, the dominant term of the nonlinear electromagnetic field is the same as that in 4-dimensional RN black holes.

Another independent differential equation of (5) depends on $h(r), h^{\prime}(r), G(r), F(r)$, and $K(\theta)$. In order to obtain consistent unknown functions, one should fix $K(\theta)=\cos (\theta)$. After simplification, one finds that second differential equation of (5) leads to

$$
e_{2}= \begin{cases}E^{\prime}(r)+\frac{E(r)}{r G(r)} \times \frac{\beta^{2}\left\{r\left[G^{\prime}(r)+F^{\prime}(r)\right]-2 F(r)\right\}}{4 E^{2}(r)+\beta^{2}}=0, & \text { ENEF, } \\ E^{\prime}(r)-\frac{E(r)}{r G(r)} \times \frac{\left(E^{2}(r)-4 \beta^{2}\right)\left\{r\left[G^{\prime}(r)+F^{\prime}(r)\right]-2 F(r)\right\}}{E^{2}(r)+4 \beta^{2}}=0, & \text { LNEF. }\end{cases}
$$

Having the electromagnetic field at hand, we are in a position to solve the gravitational field equation (4). After some cumbersome manipulation, one can show that the nonzero independent components of the field equation (4) for ENED and LNED branches are, respectively,

$$
\begin{aligned}
& e_{3}=r G^{\prime}+G-1+\Lambda r^{2}+\frac{4 \beta^{2} r^{2}}{\chi}\left(\Delta+2 \omega \Delta_{\omega}\right)=0, \\
& e_{4}=r G^{\prime \prime}+2 G^{\prime}+2 \Lambda r+\frac{8 r \beta^{2} \Delta}{\chi}=0, \\
& e_{5}=r^{2} G F^{\prime \prime}-2 F(G-1)-r F e_{4}+4 r^{2} G E^{2} \Delta_{\omega}=0,
\end{aligned}
$$

with

$$
\Delta= \begin{cases}1-e^{\omega}, & \mathrm{ENEF} \\ \ln (1-\omega), & \mathrm{LNEF}\end{cases}
$$

where $\Delta_{\omega}=-d \Delta / d \omega$ and $\omega=\chi E^{2} / 4 \beta^{2}$.

Now, we are looking for the solutions which satisfy (12) (and also (11)) with the second equation of electromagnetic field (11), simultaneously. It leads to

$$
\begin{aligned}
& G(r)=1-\frac{2 M}{r}-\frac{\Lambda r^{2}}{3}+ \begin{cases}-\frac{\beta^{2} r^{2}}{6}+\frac{\beta q}{r}\left(\int \frac{d r}{\sqrt{L_{W}}}-\int \sqrt{L_{W}} d r\right), & \text { ENEF, } \\
\frac{8 \beta^{2} r^{2}}{3}-\frac{4 q^{2}}{r} \int \frac{d r}{r^{2}(\Gamma-1)}-\frac{4 \beta^{2}}{r} \int r^{2} \ln \left(\frac{2}{\Gamma+1}\right) d r, & \text { LNEF, }\end{cases} \\
& F(r)=-G(r),
\end{aligned}
$$

where $M$ is the integration constant which is related to the ADM (Arnowitt-Deser-Misner) mass of the black hole. One may note that in Maxwell limit, $\beta \rightarrow \infty$, these slowly rotating solutions reduce to the 4-dimensional KerrNewmann black holes with slow rotation.

At this point, it is worthwhile to investigate the causal structure and physical properties of these solutions. One can show that the Kretschmann scalar $R_{\mu \nu \lambda \kappa} R^{\mu \nu \lambda \kappa}$ diverge at $r=0$ and it is finite for $r \neq 0$ and goes to $24 / l^{2}$ as $r \rightarrow \infty$. So we find that there is an essential singularity at $r=0$. Now, we look for the existence of horizons. The horizons, if any exist, are given by the zeros of the function $G(r)=$ $\left(g_{r r}\right)^{-1}$. Let us denote the largest positive root of $G(r)=0$ by $r_{+}$. Regardless of naked singularity, the black hole covers with two horizons or an extreme horizon for ordinary $\beta$. Furthermore, considering (14), one can find that for small values of the nonlinearity parameter the Schwarzschild black hole with a nonextreme horizon may be recovered. In other words, one can show that for $r \rightarrow 0$, the function $G(r)$ may be positive or negative for $\beta>\beta_{c}$ or $\beta<\beta_{c}$, respectively. The new situation appears for $\beta<\beta_{c}$, in which the black holes have one nonextreme horizon with positive temperature as 
it happens for Schwarzschild black holes (see [11] for more details).

Moreover, we can obtain some information about causal structure by considering the temperature of the black hole. By using the definition of hawking temperature on the outer horizon $r_{+}$which may be obtained through the definition of surface gravity

$$
T_{+}=\frac{1}{2 \pi} \sqrt{-\frac{1}{2}\left(\nabla_{\mu} \chi_{\nu}\right)\left(\nabla^{\mu} \chi^{\nu}\right)},
$$

where the Killing vector $\chi$ is the null generator of the event horizon. After some calculations, we find

$$
T_{+}=\frac{G^{\prime}(r)}{4 \pi}+O\left(a^{2}\right)=\frac{1-\Lambda r_{+}^{2}}{4 \pi r_{+}}+\left\{\begin{array}{lc}
\frac{\beta q\left(1-L_{W_{+}}\right)}{4 \pi r_{+} \sqrt{L_{W_{+}}}}-\frac{\beta^{2} r_{+}}{8 \pi}, & \text { ENEF } \\
\frac{q^{2}\left(2-\Gamma_{+}\right)}{\pi r_{+}^{3} \Gamma_{+}\left(\Gamma_{+}-1\right)}+\frac{\beta^{2} r_{+}}{\pi}\left(\ln \left(\frac{\Gamma_{+}^{2}-1}{2}\right)-\frac{2}{\Gamma_{+}}\right), & \text {LNEF }
\end{array}+O\left(a^{2}\right)\right.
$$

Now, we are in a position to investigate the effects of the nonlinearity parameter on the geometry of the solutions. It is interesting to mention that for the small values of the nonlinearity parameter, a new situation appears. Expanding the function $G(r)$ for large and small values of $\beta$, one can obtain

$$
\begin{aligned}
G(r)= & 1-\frac{2 M}{r}-\frac{\Lambda r^{2}}{3}+\frac{q^{2}}{r^{2}}-\frac{q^{4}}{5 \beta^{2} r^{6}} \\
& +O\left(\frac{q^{6}}{\beta^{4} r^{10}}\right), \quad \text { for large } \beta \text { or large } r
\end{aligned}
$$

Equations (18) shows that for large values of the nonlinearity parameter one can recover the RN black hole, as it should be. In addition, this equation shows that the asymptotic behavior of the solutions is AdS.

In what follows we investigate the other conserved and thermodynamics quantities. The entropy of the black hole typically satisfies the so-called area law which states that the entropy of the black hole is a quarter of the event horizon area [49-55]. This near universal law applies to almost all kinds of black holes in Einstein gravity. Since the area of the event horizon does not change up to the linear order of the rotating parameter $a$, we can easily show that the entropy of the black hole on the outer event horizon $r_{+}$can be written as

$$
S=\pi r_{+}^{2}
$$

Next, we calculate the angular momentum, the electrical charge, and the gyromagnetic ratio of these rotating nonlinear charged black holes which appear in the limit of slow rotation parameter. The angular momentum of the black hole can be calculated through the use of the quasilocal formalism of the Brown and York [56]. According to the quasilocal formalism, the quantities can be constructed from the information that exists on the boundary of a gravitating system alone. Such quasilocal quantities will represent information about the spacetime contained within the system boundary, just like the Gauss's law. In our case the stress-energy tensor can be written as

$$
T^{a b}=\frac{1}{8 \pi}\left[\Theta^{a b}-\Theta \gamma^{a b}\right]
$$

which is obtained by variation of the Lagrangian (1) with respect to $\gamma_{a b}$. To compute the angular momentum of the spacetime, one should choose a spacelike surface $\mathscr{B}$ in $\partial \mathscr{M}$ with metric $\sigma_{i j}$ and write the boundary metric in ADM form

$$
\gamma_{a b} d x^{a} d x^{a}=-N^{2} d t^{2}+\sigma_{i j}\left(d \varphi^{i}+V^{i} d t\right)\left(d \varphi^{j}+V^{j} d t\right),
$$

where the coordinates $\varphi^{i}$ are the angular variables parameterizing the hypersurface of constant $r$ around the origin and $N$ and $V^{i}$ are the lapse and shift functions, respectively. When there is a Killing vector field $\xi$ on the boundary, then the quasilocal conserved quantities associated with the stressenergy tensors of (20) can be written as

$$
Q(\xi)=\int_{\mathscr{B}} d^{n-1} x \sqrt{\sigma} T_{a b} n^{a} \xi^{b}
$$

where $\sigma$ is the determinant of the metric $\sigma_{i j}$ and $\xi$ and $n^{a}$ are the Killing vector field and the unit normal vector on the boundary $\mathscr{B}$, respectively. For boundaries with timelike $(\xi=\partial / \partial t)$ and rotational $(\varsigma=\partial / \partial \varphi)$ Killing vector fields, one obtains the quasilocal mass and angular momentum

$$
\begin{aligned}
& \mathscr{M}=\int_{\mathscr{B}} d^{n-1} x \sqrt{\sigma} T_{a b} n^{a} \xi^{b}=M, \\
& J=\int_{\mathscr{B}} d^{n-1} x \sqrt{\sigma} T_{a b} n^{a} \varsigma^{b}=M a,
\end{aligned}
$$

provided the surface $\mathscr{B}$ contains the orbits of $\varsigma$. For $a=0$, the angular momentum vanishes, and therefore $a$ is the rotational parameter of the nonlinear charged black hole. The quasilocal mass presented here is the same as ADM mass calculated by Abbott and Deser [57]. Combining (23) with (24) we get

$$
J=M a \text {. }
$$


At last, we calculate the gyromagnetic ratio of these rotating nonlinear charged black holes. One of the important subjects about the 4-dimensional charged black hole in the Einstein gravity is that it can be assigned a gyromagnetic ratio $g=2$ just like the electron in Dirac theory. Here we want to know how does the value of the gyromagnetic ratio change for slowly rotating nonlinear charged black holes in four dimensions. The magnetic dipole moment for this slowly rotating black hole is

$$
\mu=q a
$$

Therefore, the gyromagnetic ratio is given by

$$
g=\frac{2 \mu \mathscr{M}}{q J}=2
$$

which is the gyromagnetic ratio of the 4-dimensional KerrNewman black holes. Since both of the angular momenta and the magnetic dipole momenta of these black holes first appear at the linear order in rotation parameter $a$, we have led to the conclusion that the value of the gyromagnetic ratio remains $g=2$. Also, we find that the nonlinearity of electromagnetic field does not change the gyromagnetic ratio of the slowly rotating black hole.

\section{Summary and Conclusion}

In this paper, we have found a new stationary solution to gravity with NED in the presence of negative cosmological constant. This solution generalizes the corresponding static solution of [11] to include a small amount of angular momentum. Our strategy for obtaining these solutions was based on the perturbative method, where we solved the equations of motion up to the linear order in the perturbative parameter, which we chose proportional to the angular momentum. More precisely, we started from the nonrotating black hole solutions in 4 dimensions with NED [11] and considered the effect of adding a small angular momentum to the solutions. In the limit $\beta \rightarrow \infty$, the obtained solution reduces to the standard Einstein-Maxwell slowly rotating AdS black holes as expected. We calculated the mass $M$, electric charge $Q$, temperature $T$, entropy $S$, angular momentum $J$, and gyromagnetic ratio $g$ which appear up to the linear order of the angular momentum parameter $a$. Interestingly enough, we found that $\beta$ does not modify the value of the gyromagnetic ratio $g$ of the slowly rotating black holes.

In closing, we recall that this paper only considers the slow rotation approximation. One can consider second order of the angular momentum parameter to obtain smooth and precise solutions. Arbitrarily fast rotating black hole solutions with a NED are more complicated, and we have not tackled this difficult problem here. In addition, one may think about nonsingular black hole solutions of these NED theories. Let us finally mention that we have only studied fourdimensional slowly rotating black hole solutions which can be extended to $D$-dimensional nonlinear black hole solutions. This extension will appear in a forthcoming publication.

\section{Conflict of Interests}

The authors declare that there is no conflict of interests regarding the publication of this paper.

\section{Acknowledgments}

S. H. Hendi wishes to thank Shiraz University Research Council. M. Allahverdizadeh is supported by a FCT grant. This work has been supported financially by Center for Excellence in Astronomy \& Astrophysics of Iran (CEAAIRIAAM).

\section{References}

[1] A. Garcia, H. Salazar, and J. F. Plebanski, “Type-D solutions of the Einstein and Born-Infeld nonlinear-electrodynamics equations," Nuovo Cimento B, vol. 84, no. 11, pp. 65-90, 1984.

[2] M. Demianski, "Static electromagnetic geon," Foundations of Physics, vol. 16, no. 2, pp. 187-190, 1986.

[3] N. Bretón, "Born-infeld black hole in the isolated horizon framework," Physical Review D, vol. 67, no. 12, Article ID 124004, 4 pages, 2003.

[4] T. K. Dey, "Born-Infeld black holes in the presence of a cosmological constant," Physics Letters B, vol. 595, no. 1-4, pp. 484-490, 2004.

[5] H. H. Soleng, "Charged black points in general relativity coupled to the logarithmic U(1) gauge theory," Physical Review $D$, vol. 52, no. 10, pp. 6178-6181, 1995.

[6] H. P. de Oliveira, "Non-linear charged black holes," Classical and Quantum Gravity, vol. 11, no. 6, article 012, pp. 1469-1482, 1994.

[7] H. Yajima and T. Tamaki, "Black hole solutions in EulerHeisenberg theory," Physical Review D, vol. 63, no. 6, Article ID 064007, 8 pages, 2001.

[8] M. Born and L. Infeld, "Foundations of the new field theory," Proceedings of the Royal Society A, vol. 144, no. 852, pp. 425-451, 1934.

[9] I. H. Salazar, D. A. García, and J. Plebański, "Duality rotations and type D solutions to Einstein equations with nonlinear electromagnetic sources," Journal of Mathematical Physics, vol. 28, no. 9, pp. 2171-2181, 1987.

[10] S. H. Hendi, "Asymptotic charged BTZ black hole solutions," Journal of High Energy Physics, vol. 2012, no. 3, article 65, 2012.

[11] S. H. Hendi, "Asymptotic Reissner-Nordström black holes," Annals of Physics, vol. 333, pp. 282-289, 2013.

[12] S. H. Hendi and A. Sheykhi, "Charged rotating black string in gravitating nonlinear electromagnetic fields," Physical Review $D$, vol. 88, Article ID 044044, 2013.

[13] I. Z. Stefanov, S. S. Yazadjiev, and M. D. Todorov, "Scalartensor black holes coupled to Euler-Heisenberg nonlinear electrodynamics," Modern Physics Letters A: Particles and Fields, Gravitation, Cosmology, Nuclear Physics, vol. 22, no. 17, pp. 12171231, 2007.

[14] L. Hollenstein and F. S. N. Lobo, "Exact solutions of $f(R)$ gravity coupled to nonlinear electrodynamics," Physical Review D, vol. 78, Article ID 124007, 2008.

[15] H. A. Gonzalez, M. Hassaine, and C. Martinez, "Thermodynamics of charged black holes with a nonlinear electrodynamics source," Physical Review D, vol. 80, Article ID 104008, 2009. 
[16] O. Miskovic and R. Olea, "Quantum statistical relation for black holes in nonlinear electrodynamics coupled to Einstein-GaussBonnet AdS gravity," Physical Review D, vol. 83, Article ID 064017, 2011.

[17] J. Diaz-Alonso and D. Rubiera-Garcia, “Thermodynamic analysis of black hole solutions in gravitating nonlinear electrodynamics," General Relativity and Gravitation, vol. 45, no. 10, pp. 1901-1950, 2013.

[18] S. H. Mazharimousavi, M. Halilsoy, and O. Gurtug, "A new Einstein-nonlinear electrodynamics solution in $2+1$ dimensions," The European Physical Journal C, vol. 74, article 2735, 2014.

[19] D. H. Delphenich, "Nonlinear electrodynamics and QED," http://arxiv.org/abs/hep-th/0309108.

[20] D. H. Delphenich, "Nonlinear optical analogies in quantum electrodynamics," http://arxiv.org/abs/hep-th/0610088.

[21] J. Schwinger, "On gauge invariance and vacuum polarization," Physical Review, vol. 82, pp. 664-679, 1951.

[22] W. Heisenberg and H. Euler, "Consequences of dirac theory of the positron," Zeitschrift für Physik, vol. 98, pp. 714-732, 1936, Translation by: W. Korolevski and H. Kleinert.

[23] H. Yajima and T. Tamaki, "Black hole solutions in EulerHeisenberg theory," Physical Review D, vol. 63, no. 6, Article ID 064007, 2001.

[24] V. A. de Lorenci and M. A. Souza, "Electromagnetic wave propagation inside a material medium: an effective geometry interpretation," Physics Letters B, vol. 512, no. 3-4, pp. 417-422, 2001.

[25] V. A. De Lorenci and R. Klippert, "Analogue gravity from electrodynamics in nonlinear media," Physical Review D, vol. 65, no. 6, Article ID 064027, 2002.

[26] M. Novello and E. Bittencourt, "Gordon metric revisited," Physical Review D, vol. 86, Article ID 124024, 2012.

[27] M. Novello, S. Perez Bergliaffa, J. Salim, V. A. de Lorenci, and R. Klippert, "Analogue black holes in flowing dielectrics," Classical and Quantum Gravity, vol. 20, no. 5, pp. 859-871, 2003.

[28] H. J. Mosquera Cuesta and J. M. Salim, "Non-linear electrodynamics and the gravitational redshift of highly magnetized neutron stars," Monthly Notices of the Royal Astronomical Society, vol. 354, no. 4, pp. L55-L59, 2004.

[29] H. J. M. Cuesta and J. M. Salim, "Nonlinear electrodynamics and the surface redshift of pulsars," Astrophysical Journal Letters, vol. 608, no. 2, pp. 925-929, 2004.

[30] Z. Bialynicka-Birula and I. Bialynicki-Birula, "Nonlinear effects in quantum electrodynamics: photon propagation and photon splitting in an external field," Physical Review D, vol. 2, no. 10, pp. 2341-2345, 1970.

[31] E. Ayón-Beato and A. Garcia, "Non-singular charged black hole solution for non-linear source," General Relativity and Gravitation, vol. 31, no. 5, pp. 629-633, 1999.

[32] E. Ayón-Beato and A. García, "New regular black hole solution from nonlinear electrodynamics," Physics Letters B, vol. 464, no. 1-2, pp. 25-29, 1999.

[33] V. A. De Lorenci, R. Klippert, M. Novello, and J. M. Salim, "Nonlinear electrodynamics and FRW cosmology", Physical Review D, vol. 65, no. 6, Article ID 063501, 2002.

[34] I. Dymnikova, "Regular electrically charged vacuum structures with de Sitter centre in nonlinear electrodynamics coupled to general relativity," Classical and Quantum Gravity, vol. 21, no. 18, pp. 4417-4428, 2004.
[35] C. Corda and H. J. Mosquera Cuesta, "Removing black hole singularities with nonlinear electrodynamics," Modern Physics Letters A. Particles and Fields, Gravitation, Cosmology, Nuclear Physics, vol. 25, no. 28, pp. 2423-2429, 2010.

[36] C. Corda and H. J. Mosquera Cuesta, "Inflation from R2 gravity: a new approach using nonlinear electrodynamics," Astroparticle Physics, vol. 34, no. 7, pp. 587-590, 2011.

[37] J. Plebanski, Lectures on Non-Linear Electrodynamics, Nordita, 1968.

[38] S. Mignemi and N. R. Stewart, "Dilaton-axion hair for slowly rotating Kerr black holes," Physics Letters B, vol. 298, no. 3-4, pp. 299-304, 1993.

[39] M. S. Volkov and N. Straumann, "Slowly rotating non-abelian black holes," Physical Review Letters, vol. 79, no. 8, pp. 1428-1431, 1997.

[40] A. N. Aliev, "A slowly rotating charged black hole in five dimensions," Modern Physics Letters A, vol. 21, no. 9, pp. 751757, 2006.

[41] A. N. Aliev, "Gyromagnetic ratio of charged Kerr-anti-de Sitter black holes," Classical and Quantum Gravity, vol. 24, no. 18, pp. 4669-4677, 2007.

[42] T. Ghosh and S. SenGupta, "Slowly rotating dilaton black hole in anti-de Sitter spacetime," Physical Review D, vol. 76, no. 8, 087504, 4 pages, 2007.

[43] H. C. Kim and R. G. Cai, "Slowly rotating charged GaussBonnet black holes in AdS spaces," Physical Review D, vol. 77, no. 2, Article ID 024045, 2008.

[44] A. Sheykhi and M. Allahverdizadeh, "Higher dimensional slowly rotating dilaton black holes in AdS spacetime," Physical Review D: Particles, Fields, Gravitation, and Cosmology, vol. 78, no. 6, Article ID 064073, 6 pages, 2008.

[45] S. H. Hendi, "Slowly rotating black holes in Einstein-generalized maxwell gravity," Progress of Theoretical Physics, vol. 124, pp. 493-502, 2010.

[46] A. N. Aliev, "Rotating black holes in higher dimensional Einstein-Maxwell gravity," Physical Review D, vol. 74, Article ID 024011, 2006.

[47] M. Abramowitz and I. A. Stegun, Handbook of Mathematical Functions, Dover, New York, NY, USA, 1972.

[48] R. M. Corless, G. H. Gonnet, D. E. G. Hare, D. J. Jeffrey, and D. E. Knuth, "On the Lambert $W$ function," Advances in Computational Mathematics, vol. 5, no. 1, pp. 329-359, 1996.

[49] J. D. Bekenstein, "Black holes and the second law," Lettere Al Nuovo Cimento, vol. 4, no. 15, pp. 737-740, 1972.

[50] J. D. Bekenstein, "Black holes and entropy," Physical Review D, vol. 7, p. 2333, 1973.

[51] S. W. Hawking and C. J. Hunter, "Gravitational entropy and global structure," Physical Review D, vol. 59, no. 4, Article ID 044025, 10 pages, 1999.

[52] S. W. Hawking, C. J. Hunter, and D. N. Page, "NUT charge, antide Sitter space, and entropy," Physical Review. D, vol. 59, no. 4, Article ID 044033, 6 pages, 1999.

[53] R. B. Mann, "Misner string entropy," Physical Review D, vol. 60, Article ID 104047, 1999.

[54] R. B. Mann, "Entropy of rotating Misner string spacetimes," Physical Review D, vol. 61, no. 8, Article ID 084013, 6 pages, 2000.

[55] C. J. Hunter, "Action of instantons with a nut charge," Physical Review D, vol. 59, no. 2, 1999. 
[56] J. D. Brown and J. York, "Quasilocal energy and conserved charges derived from the gravitational action," Physical Review D, vol. 47, no. 4, pp. 1407-1419, 1993.

[57] L. F. Abbott and S. Deser, "Stability of gravity with a cosmological constant," Nuclear Physics B, vol. 195, no. 1, pp. 76-96, 1982. 

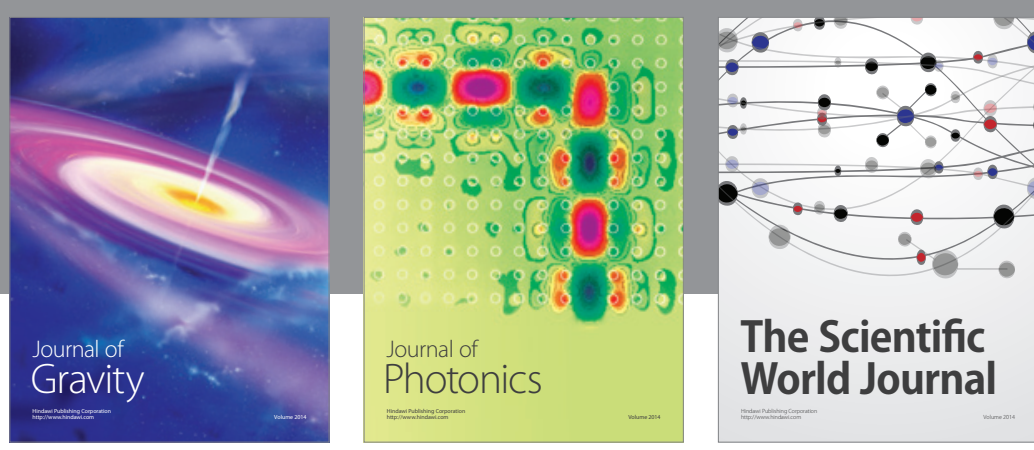

The Scientific World Journal
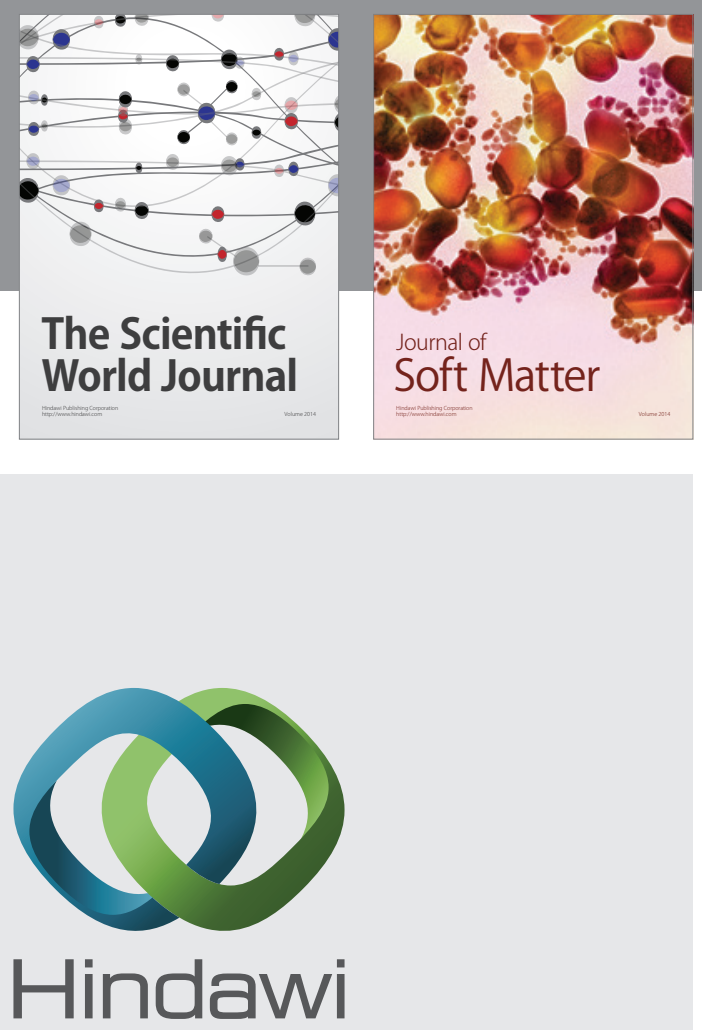

Submit your manuscripts at

http://www.hindawi.com

nternational Journal of

Statistical Mechanics
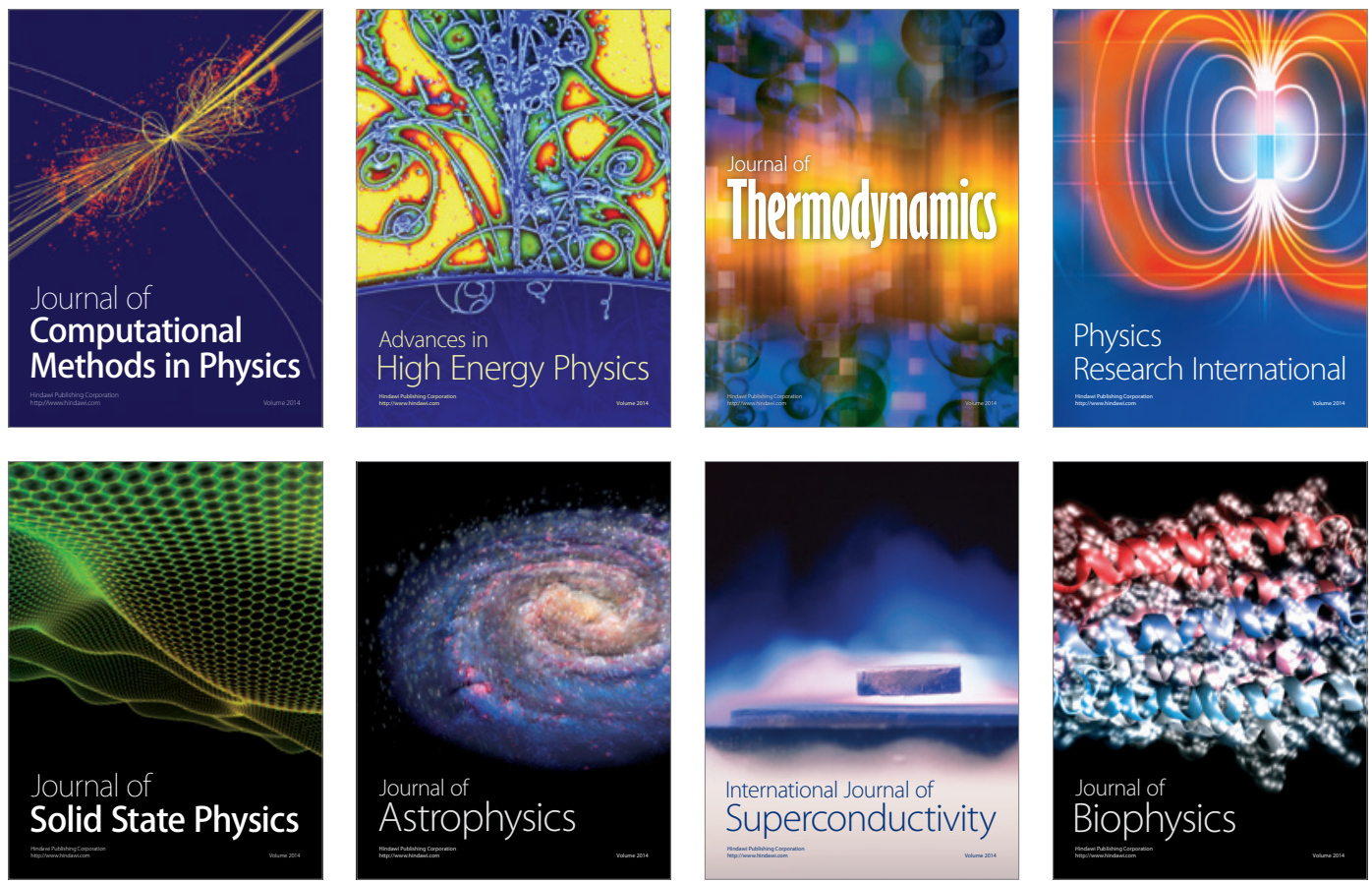
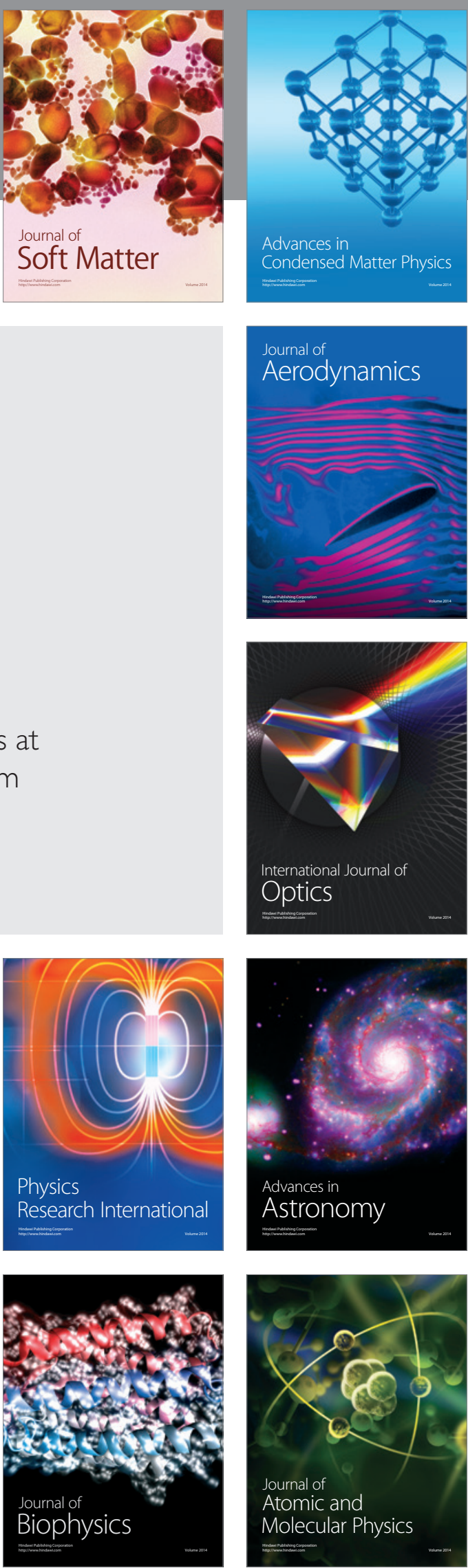